\section{Postoperative Recurrence of Trigeminal Neuralgia with Brown Spots on the Nerve Root}

Sir,

Trigeminal neuralgia (TN) is a common disease in functional neurosurgery. Microvascular decompression (MVD) is the most effective treatment. More than $10 \%$ of patients with good immediate postoperative relief has recurrent pain one year after surgery, ${ }^{1}$ and the exact cause is still unclear. A recurrent case as encountered in this department having dark brown spots on the nerve root, which is very rare.
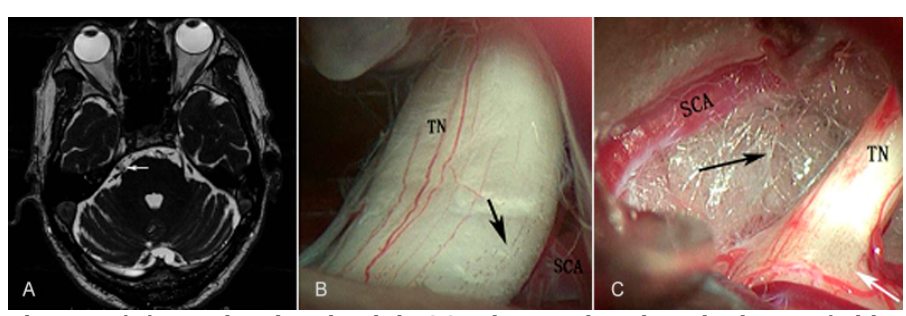

Figure 1: (A) MRI showing the right SCA close to the trigeminal nerve (white arrow: SCA; black arrow: TN). (B) Trigeminal nerve root was compressed by the SCA and covered with dark brown spots (black arrow: dark brown spots). (C) The artery and nerve were separated with Teflon felt (white arrow: dark brown spots; black arrow: Teflon felt).

SCA:Superiorcerebellarartery, TN:Trigeminalnerve.

A 65-year man was admitted to the Hospital with facial pain on the right side, at the V2 and V3 distribution for two years. Magnetic resonance imaging (MRI) showed that the right superior cerebellar artery (SCA) was close to the trigeminal nerve root (Figure 1A). His medical history was unremarkable. The MVD operation was performed through retrosigmoid it is keyhole approach. During the operation, we found that the trigeminal nerve root was compressed by the SCA and covered with dark brown spots (Figure 1B). The artery and nerve was separated with Teflon felt (Figure 1C). The patient recovered well after surgery and the pain disappeared completely. The pain recurred in the V2 distribution 14 months after surgery. The radiofrequency thermal rhizolysis was performed and the pain relieved again.

The exact cause of postoperative recurrence of trigeminal neuralgia (TN) is still unclear and it is an important issue that plagues surgeons. Jianghua et al. ${ }^{2}$ described the recurrence of TN with the appearance of brown spots on the trigeminal nerve root. No other similar cases were reported. The special aspect of this relapsed patient is the presence of brown spots on the trigeminal nerve root, similar to the spotted mature banana skin, in sharp contrast to the smooth surface of normal nerve root. The authors speculate that it may be a manifestation of neurodegeneration, and may increase the susceptibility and recurrence risk of patients to trigeminal neuralgia. More cases and studies are needed to explore what these brown spots are and how they contribute to the recurrence of trigeminal neuralgia.

\section{CONFLICT OF INTEREST:}

Authors declared no conflict of interest.

\section{AUTHORS' CONTRIBUTION:}

WT, ZW, XM, JZ, PY: Contributed to the design of the work; drafted the work and revised it critically for important intellectual content; approved the final version to be published.

\section{REFERENCES}

1. Oesman C, Mooij JJ. Long-term follow-up of microvascular decompression for trigeminal neuralgia. Skull Base 2011; 21:313-22.

2. Jianghua Z, Yanhua Z, Chong W. Observation of the curative effect of MVD and its modified operation on trigeminal neuralgia with brown spots of nerve root. Chin J Neurotrauma Surg 2017; 3:268-71.

Wei Tian ${ }^{1}$, Zhuangzhi Wang ${ }^{2}$, Xin Meng ${ }^{1}$, Jidian Zou ${ }^{1}$ and Pingping $Y u^{1}$

${ }^{1}$ Department of Neurosurgery, Weihai Central Hospital, The Affiliated Hospital of Qingdao University Medical College, Weihai City, China

${ }^{2}$ Department of Rehabilitation Medicine, Weihai Central Hospital, The Affiliated Hospital of Qingdao University Medical College, Weihai City, China

Correspondence to: Pingping Yu, West No. 3, Mishan Dong Road, Wendeng District, Weihai City, Shandong Province, China

E-mail: yupingping52@163.com

Received: February 21, 2020; Revised: May 03, 2020;

Accepted: May 03, 2020

DOI: https://doi.org/10.29271/jcpsp.2020.05.555 